\title{
First record of Achillea alpina L. subsp. pulchra (Koidz.) Kitam. (Asteraceae) in Korea
}

\author{
Eun-Mi Sun, Hyoung-Tak Im¹, Hyuk-Jin Kim, Dong Chan Son, \\ Kang Hyup Lee and Kyung Choi* \\ Forest Biodiversity Division, Korea National Arboretum, Pocheon 11186, Korea \\ ${ }^{1}$ Department of Biology, Chonnam National University, Gwangju, 61186, Korea \\ (Received 20 March 2017; Revised 25 April 2017; Accepted 19 June 2017)

\section{한국 미기록 식물: 갯톱풀(국화과) \\ 선은미 · 임형탁 ${ }^{1} \cdot$ 김혁진 · 손동찬 · 이강협 · 최경* 국립수목원 산림생물조사과, ${ }^{1}$ 전남대학교 자연과학대학 생물학과}

\begin{abstract}
Achillea alpina L. subsp. pulchra (Koidz.) Kitam., an unrecorded subspecies belonging to the genus Achillea of Asteraceae, was discovered and is illustrated here. It is known as an endemic species of Hokkaido, Japan, but we discovered it along the seashore at Uljin in Gyeongsangbuk-do, Korea. Having a relatively equally serrate or dentate leaf margin and sparsely villous involucres, this subspecies is easily distinguished from its related taxa. Based on its habitat, it was termed Gaet-top-pul in Korean. We provide a description, illustrations, photographs and a key of related taxa in Korea.
\end{abstract}

Keywords: Achillea, new record taxa, Achillea alpina L. subsp. pulchra (Koidz.) Kitam.

적 요: 국화과 톱풀속의 미기록 식물인 갯톱풀(Achillea alpina L. subsp. pulchra (Koidz.) Kitam.)을 발견하여 기재하고 도해하였다. 갯톱풀은 일본 특산으로 홋카이도 해안가에 자생한다고 알려져 있으나 경상남도 울진 군 일대의 해안가에서 분포를 확인하였다. 갯톱풀은 엽연이 비교적 고른 치아상거치 또는 예거치이고, 잎과 총포에 털이 성기어 다른 톱풀속 식물들과 쉽게 구별된다. 국명은 미기록 식물의 자생지를 고려하여 '갯톱풀'이 라 신칭하였고, 이에 대한 기재, 그림도해, 사진 및 검색표를 제시하였다.

주요어: 톱풀속, 미기록 식물, 갯톱풀

The genus Achillea (Asteraceae) is composed of approximately 110-140 species (El-Shazly et al., 2004; Saukel et al., 2003; Guo et al., 2005; Ehrendorfer and Guo, 2006), and grows throughout the northern hemisphere (Davis, 1975). Three species of Achillea are known in Korea, A. alpina L., A. acuminata (Ledeb.) Sch. Bip. and A. millefolium L. A. millefolium is easily distinguished by having 3-pinnatisect leaves from the other two Korean species with 2-pinnatisect leaves. A. acuminata is distributed mainly in east Siberia, Mongolia, Manchuria and northeast part of Korean Peninsula. Otherwise, A. alpina is widely distributed from India to Japan, and has vast morphological variation range. And so, it has been divided into 3 infraspecific taxa in Korea, A. alpina L. subsp. alpina var. alpina, subsp. alpina var. discoidea (Regel) Kitam., and subsp. rhodoptarmica (Nakai) Kitam.

We found A. alpina L. subsp. pulchra (Koidz.) Kitam. in

\footnotetext{
*Author for correspondence: kchoi@korea.kr
} 


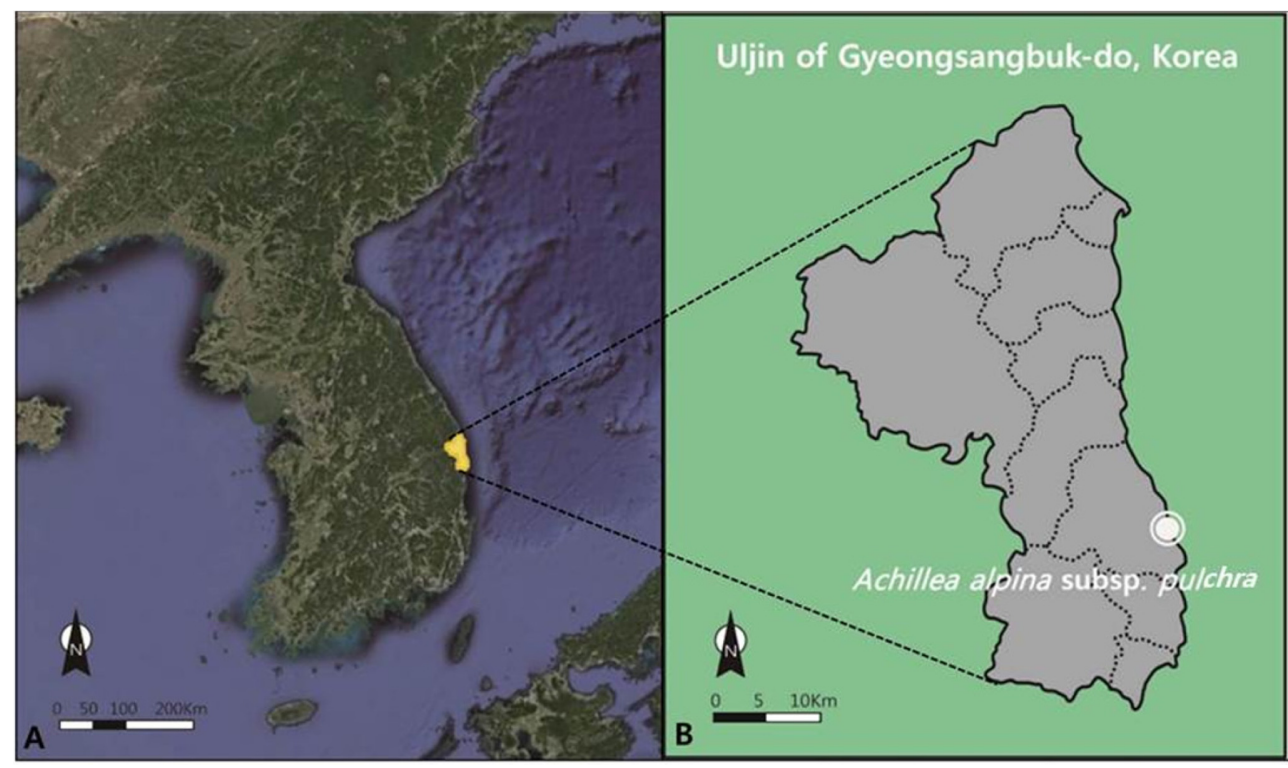

Fig. 1. Location of the first record of Achillea alpina subsp. pulchra. A. Gyeongsangbuk-do (yellow) in South Korea. B. Collection area of Gyeongsangbuk-do (gray).

Gyeongsangbuk-do, Uljin during the field investigation in 2014 (Fig. 1). This taxon was reported as a new species from coastal region of Hokkaido, Japan by Koidzumi (1918), and later it was retreated taxonomically by Kitamura (1968) as A. alpina subsp. pulchra (comb. et stat. nov). It has been considered as an endemic of Hokkaido, Japan (Hara, 1938; Koyama, 1995). We provide the illustration (Fig. 2) and photographs of $A$. alpina subsp. Pulchra (Fig. 3).

\section{Taxonomic Treatment}

Achillea alpina L. subsp. pulchra (Koidz.) Kitam., Acta Phytotax. Geobot. 23: 4, 1968; A. pulchra Koidzumi, Bot. Mag. Tokyo 32: 59, 1918.

Perennial herbs. Rhizomes creeping. Stem erect, $60-80 \mathrm{~cm}$ high, branched in upper part, loosely villous, glandular. Leaves minutely toothed or serrated lobed, loosely or sparsely villous, color green. Basal and lower leaves withered at anthesis. Middle leaves sessile, blade, oblong-linear, 5-9 cm long, 9$13 \mathrm{~mm}$ wide, base attenuate, margin relatively equally serrate or dentate, apex acute to obtuse, pellucidly dotted; leaf teeth acute, cartilaginous cuspidate. Inflorescence corymb; erect, terminal, Capitula semi-globose, $9-12 \mathrm{~mm}$ in diameter, loosely villous; Involucre $4.5-5 \mathrm{~mm}$ wide, $5-5.5 \mathrm{~mm}$ long, imbricate, green, with prominent midrib, in 3-series; outer involucal bract short, ovate-oblong, middle involucal bract oblong to lanceolate, inner involucal bract linear-lanceolate; in middle, green, with prominent midrib; palea oblanceolate; Ligulate florets 7-8, 7-9 mm long, with isolated, ligules white, oval, 3$4 \mathrm{~mm}$ long, $2.5-3.5 \mathrm{~mm}$ wide, shallowly 3 roundish lobed at apex, tube pellucidly dotted; Disk florets 25-40, bisexual, white, tubular, 5-lobed, 3-5 mm long, glandular. Stamens 5, syngenesious. Achenes oblanceolate, 2-2.5 mm long, 1$1.5 \mathrm{~mm}$ wide, winged, wing somewhat thick, brown, pappus absent. Flowering: Jul to Aug. Fruiting: Sep to Oct.

Specimen examined: KOREA. Gyeongsangbuk-do: Uljin, $36^{\circ} 50^{\prime} 06.84^{\prime \prime} \mathrm{N}, 129^{\circ} 26^{\prime} 31.53^{\prime \prime}$, elev. 15 m, 1 Sep 2016, E. M. Sun and H. S. Kim, SEM201600831001 (KH).

Notes: Four infraspecific taxa of $A$. alpina are distinguished each other in some morphological characters; incised kinds of leaf margin, wide of head, density of hairs on involucre, diameter of involucre, and length of ligulate tube, etc. (Table 1). A. alpina subsp. alpina var. alpina and subsp. alpnia var. discoidea have pinnately cleft margin, densely villous hair on involucre (Fig. 4A, B) and short (1.5 mm long) ligulate tube. Between above two varieties, subsp. alpnia var. discoidea is characterized by having smaller leaves (4-6 cm long, 5-7 mm wide), smaller heads (4-4.5 $\mathrm{mm}$ in diameter), smaller involucre (4-5 mm long, 3-4 mm wide) and shorter ligule limb (1 mm long) (Fig. 4B) than those of subsp. alpnia var. discoidea. Otherwise, A. alpina subsp. pulchra and subsp. rhodoptarmica have dentately or serrately lobed margin, loosely villous hair on involucre (Fig. 4C) and long ligulate tube (2-2.5 mm long) (Fig. 5C). And they are distinguished easily from each other by floret colors and habitats. A. alpina subsp. rhodoptarmica 
B

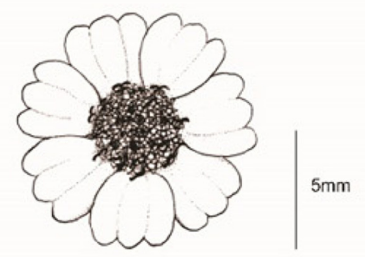

C
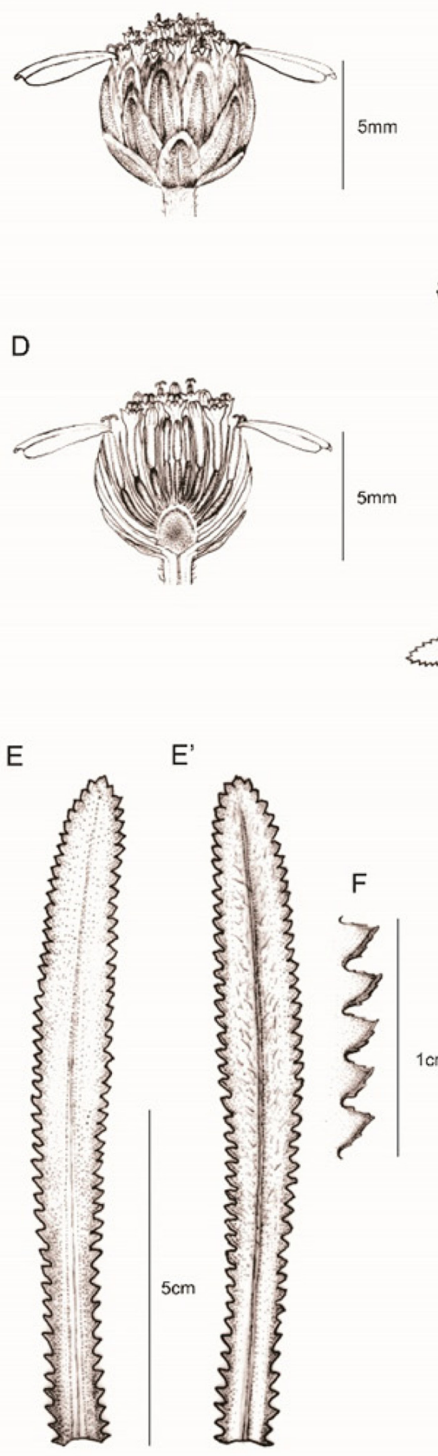

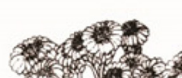

198.
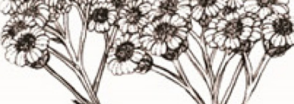

.

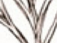

V

(

s.s
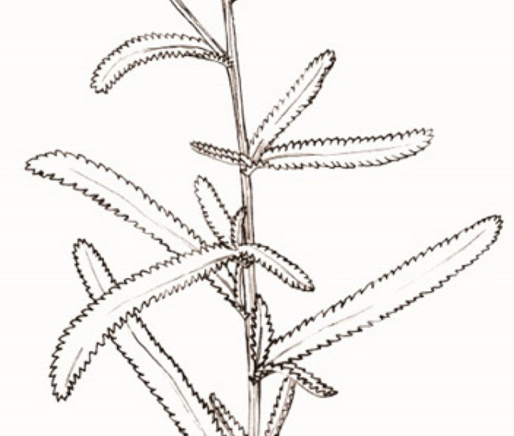

$\mathrm{H}$
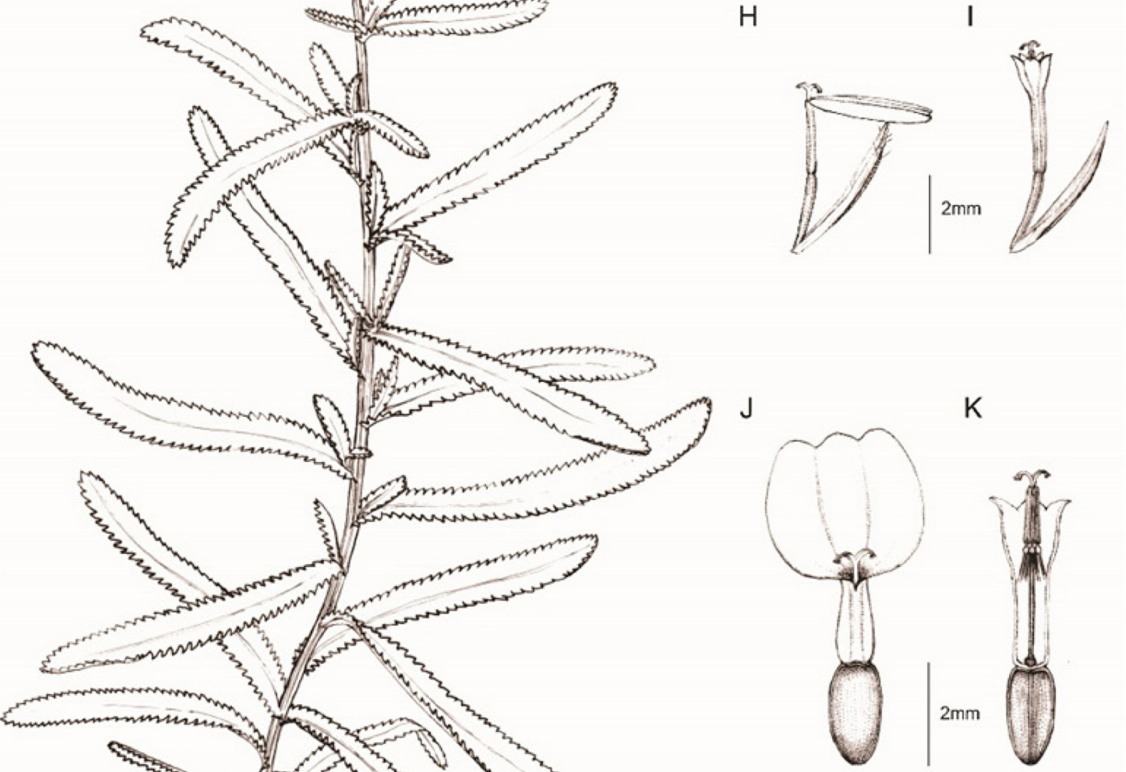

A

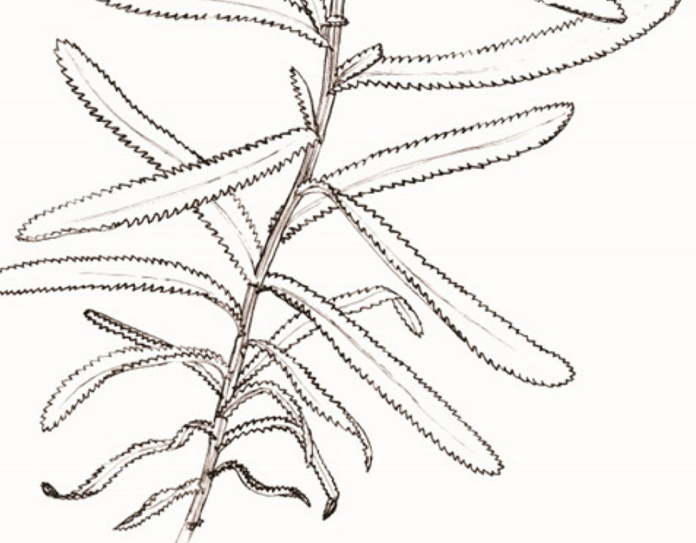

L

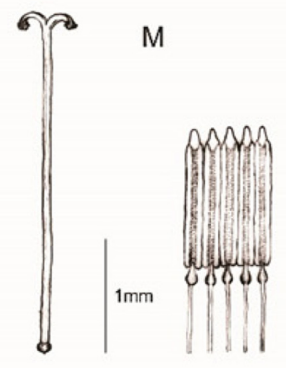

$\mathrm{N}$

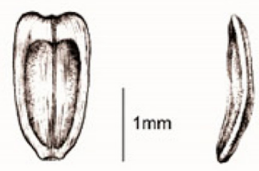

Fig. 2. Achillea alpina subsp. pulchra. A. Plant. B-D. Capitulum. E. Leaf adaxial side. E'. Leaf abaxial side. F. Cartilaginous leaf teeth. G. Bracts of involucre and palea (rightnost). H. Ligulate floret and involucral bract. I. Disk floret and palea. J. Ligulate floret. K. Disk floret. L. Style. M. Anther. N. Achene. 


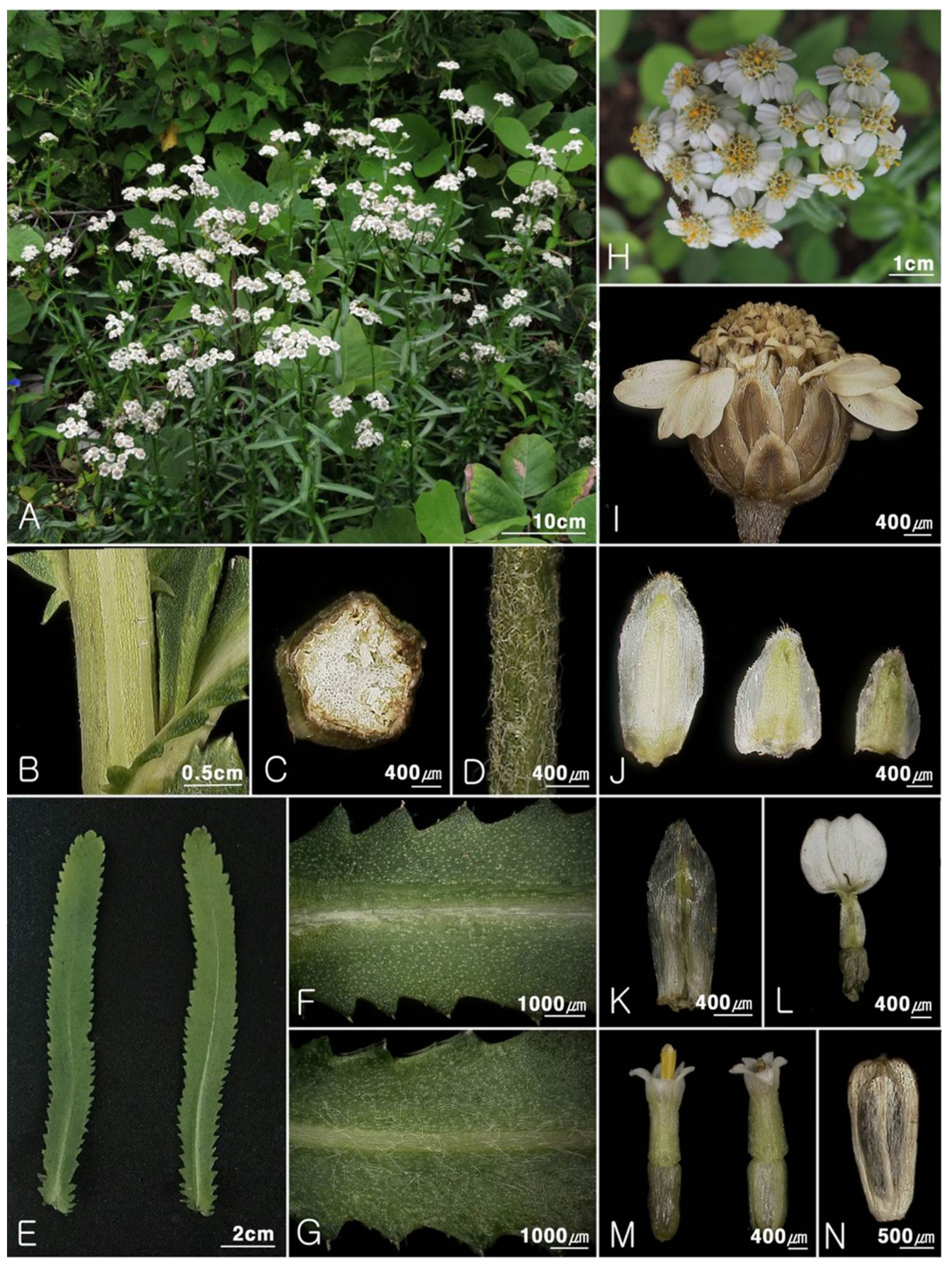

Fig. 3. Achillea alpina subsp. pulchra. A. Habit. B. Stem. C. Cross section of stem. D. Peduncle. E. Leaf. F. Leaf adaxial surface. G. Leaf abaxial surface. H. Corymb. I. Capitula. J. Bract of involucre. K. Palea. L. Ligulate floret. M. Disk floret. N. Achene. 

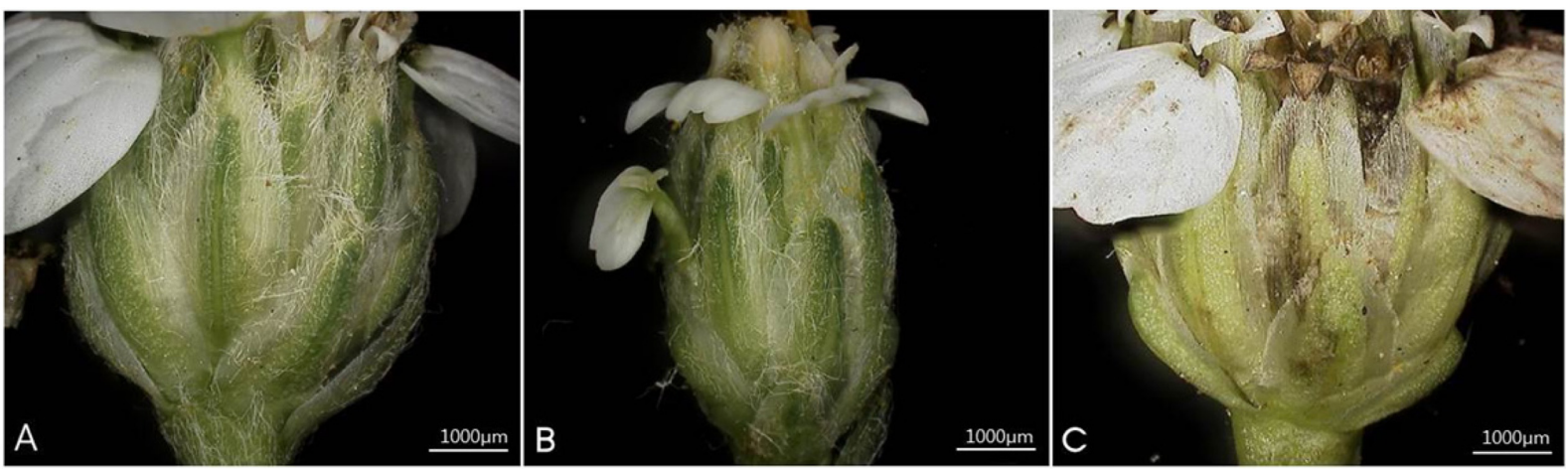

Fig. 4. Involucres of Achillea alpina. A. A. alpina subsp. alpina var. alpina. B. A. alpina subsp. alpnia var. discoidea. C. A. alpina subsp. pulchra.

Table 1. Comparison of morphological characters between Achillea alpina subsp. pulchra and its related taxa.

\begin{tabular}{|c|c|c|c|c|c|}
\hline \multicolumn{2}{|l|}{ Characters } & $\begin{array}{l}\text { A. alpina subsp. } \\
\text { alpina var. alpina }\end{array}$ & $\begin{array}{l}\text { A. alpina subsp. alpina var. } \\
\text { discoidea }\end{array}$ & $\begin{array}{l}\text { A. alpina subsp. } \\
\text { pulchra }\end{array}$ & $\begin{array}{l}\text { A. alpina subsp. } \\
\text { rhodoptarmica }\end{array}$ \\
\hline \multicolumn{2}{|l|}{ Habits } & Perennial & Perennial & Perennial & Perennial \\
\hline \multicolumn{2}{|l|}{ Habitat } & Mountain & Mountain & Seashores & Mountain \\
\hline \multicolumn{2}{|c|}{ Plant height $(\mathrm{cm})$} & $30-80$ & $30-80$ & $60-80$ & $50-80$ \\
\hline \multirow[t]{5}{*}{ Leaf blade } & shape & Linear-lanceolate & Linear-lanceolate & Linear-lanceolate & Linear-lanceolate \\
\hline & length $(\mathrm{cm})$ & $6-10$ & $4-6$ & $5-9$ & $5-6$ \\
\hline & width (mm) & $7-17$ & $5-7$ & $9-13$ & $5-8$ \\
\hline & apex & Acute to obtuse & Acute to obtuse & Acute to obtuse & Acuminate \\
\hline & margin & Bi-pinnately cleft & $\begin{array}{l}\text { Bi-pinnately cleft } \\
\text { or dentately cleft }\end{array}$ & Dentate or serrate & Dentate or serrate \\
\hline Capitulum & diameter $(\mathrm{mm})$ & $7-9$ & $4-4.5$ & $9-12$ & $6-7$ \\
\hline \multirow[t]{5}{*}{ Involucre } & shape & Semi-globose & Campanulate & Semi-globose & Semi-globose \\
\hline & hair & Densely villous & Densely villous & Loosely villous & Loosely villous \\
\hline & length (mm) & 5 & $4-5$ & $5-5.5$ & $4-5$ \\
\hline & width (mm) & $4-5(7)$ & $3-4$ & $4.5-5.5$ & $3-4$ \\
\hline & row & 3 & 3 & 3 & 3 \\
\hline \multirow[t]{7}{*}{ Ligulate floret } & colour & White & White & White & Red or pink \\
\hline & number & $5-7$ & $5-7$ & $7-8$ & - \\
\hline & length (mm) & $4-4.5$ & $<3$ & $3-4$ & $4-5$ \\
\hline & width (mm) & $2.5-3$ & 1.5 & $2.5-3.5$ & $2.7-3.2$ \\
\hline & apex & 3-lobed & 3-lobed & 3-lobed & 3-lobed \\
\hline & limb length $(\mathrm{mm})$ & $3-4.5$ & 1 & $2.5-4$ & $2-2.5$ \\
\hline & tube $(\mathrm{mm})$ & 1.5 & 1.5 & $2-2.5$ & $2-2.5$ \\
\hline \multirow[t]{2}{*}{ Disk floret } & apex & 5-lobed & 5-lobed & 5-lobed & 5-lobed \\
\hline & length (mm) & $2-3$ & $2-2.5$ & $3-5$ & 2.5 \\
\hline \multirow[t]{2}{*}{ Achene } & length (mm) & $2-3$ & $2-2.5$ & $2-2.5$ & $2-2.5$ \\
\hline & width (mm) & $1-1.2$ & 1 & $1-1.5$ & 1 \\
\hline
\end{tabular}




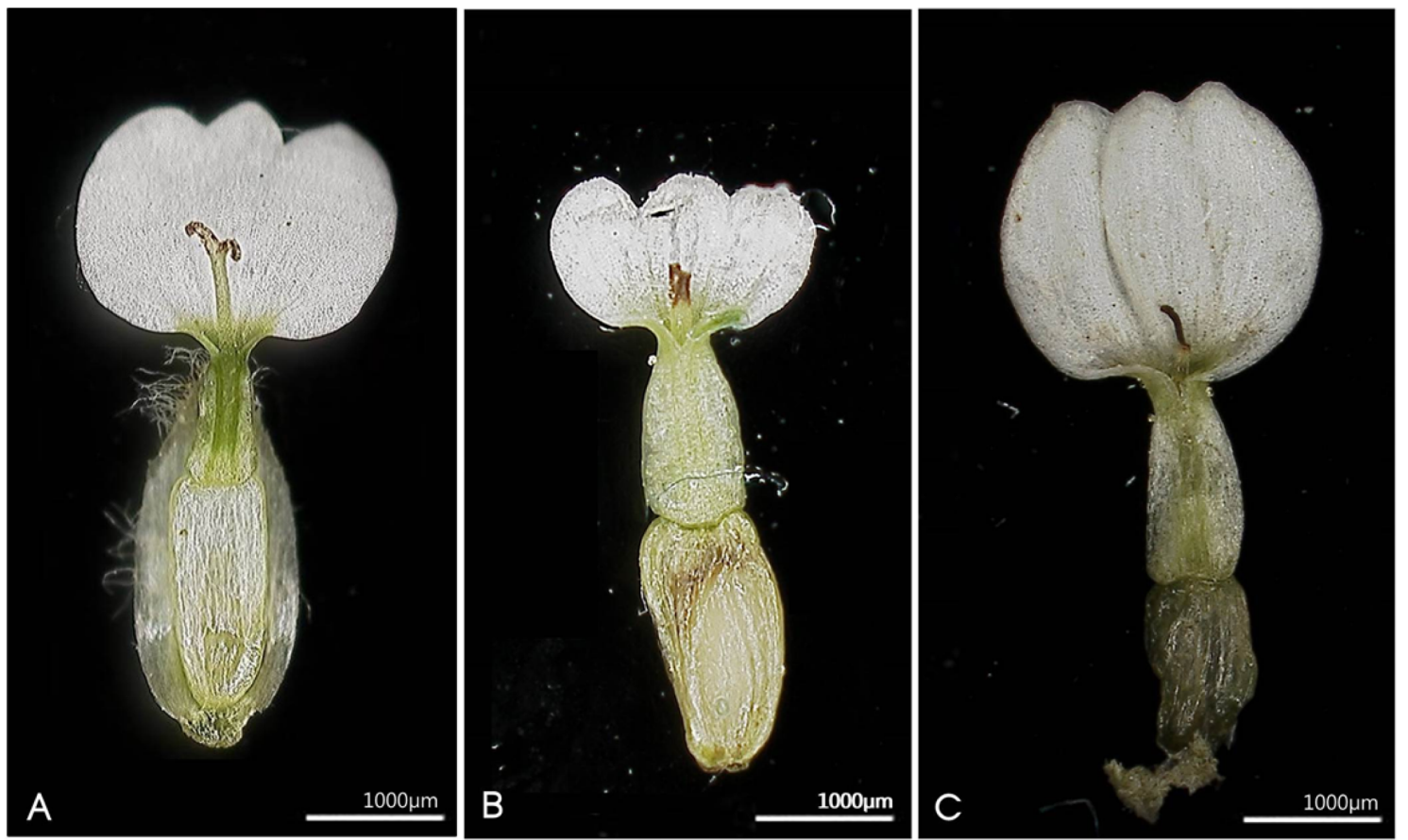

Fig. 5. Ligulate floret of Achillea alpina. A. A. alpina subsp. alpina var. alpina. B. A. alpina subsp. alpnia var. discoidea. C. A. alpina subsp. pulchra.

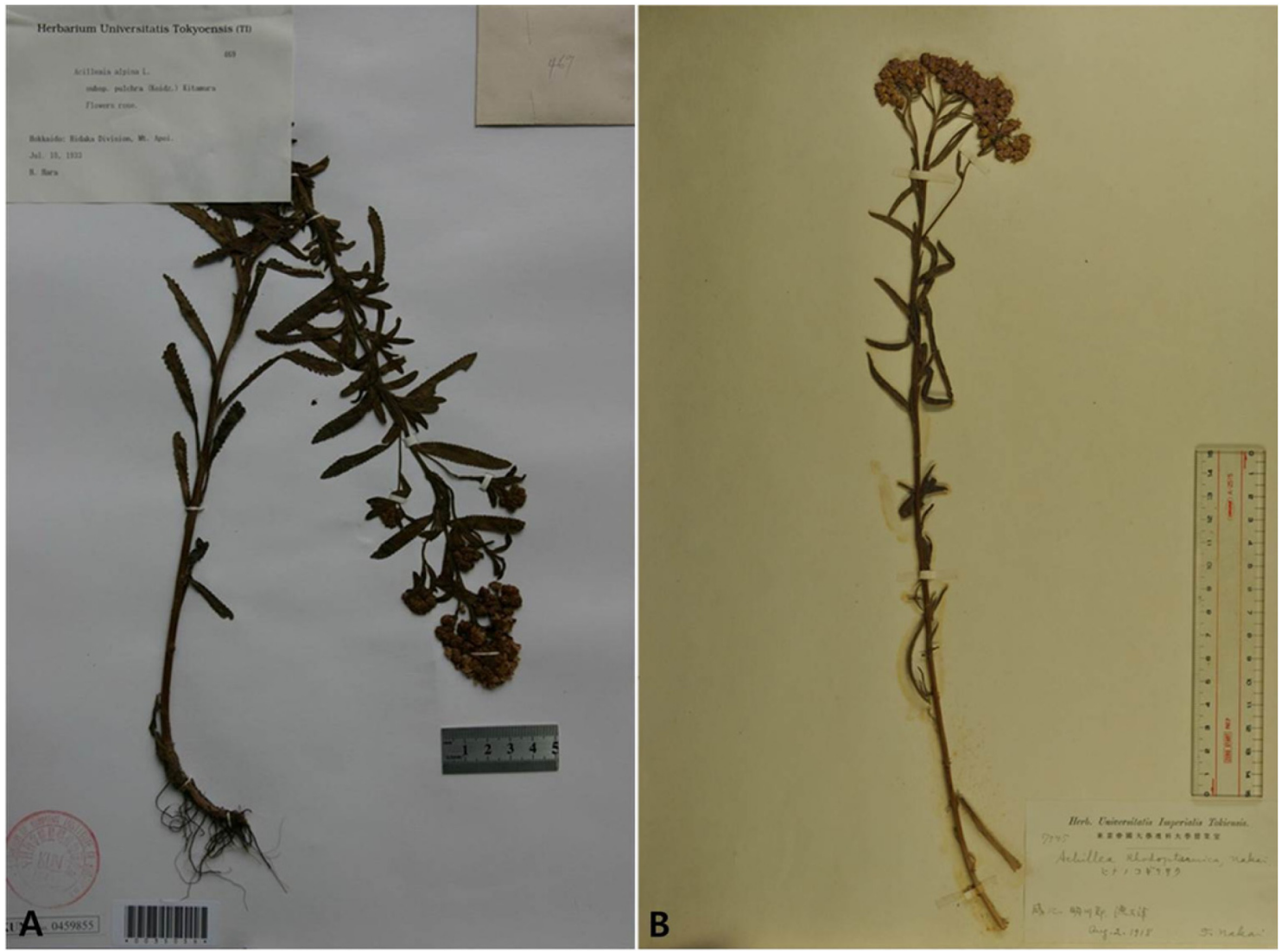

Fig. 6. Digital images of specimens of Achillea alpina in the University of Tokyo (TI). A. A. alpina subsp. pulchra. B. A. alpina subsp. rhodoptarmica. 
has red or pink florets and is distributed only in mountainous region of Gilju and Myeongcheon, Hamgyeongbuk-do (Im, 1999). Because there was no specimen of subsp. pulchra and subsp. rhodoptarmica in any herbarium of Korea, its morphological characters were gathered from digital images of specimens deposited in the University of Tokyo (TI) (Fig. 6) and literatures (Koidzumi, 1918; Kitamura, 1968). Otherwise subsp. pulchra has white florets and is distributed in coastal area of Gyeongsangbuk-do, Uljin.

\section{Key to A. alpina subsp. pulchra and its related taxa in Korea}

1. Leaves pinnately to parted, segments linear

A. alpina subsp. alpina var. alpina

2. Capitula campanulate or elliptic, ligulate florets less than $3 \mathrm{~mm}$ long

A. alpina subsp. alpina var. discoidea

2. Capitula semi-globose, ligulate florets more than $3 \mathrm{~mm}$ long

1. Leaves minutely dentate or serrated-lobed, segments acute

3. Ligulate florets white …... A. alpina subsp. pulchra

3. Ligulate florets red or pink

A. alpina subsp. rhodoptamica

\section{Acknowledgments}

This research was supported by a grant from Korea Endemic Research (1900-1935-300-220-01) of the Korea National Arboretum. We are deeply indebted to Heesu Kim.

\section{Literature Cited}

Davis, P. H. 1975. Flora of Turkey and the East Aegean Islands. Vol. 5. Edinburgh University Press, Edinburgh, 890 pp.

Ehrendorfer, F. and Y.-P. Guo. 2006. Multidisciplinary studies on Achillea sensu lato (Compositae-Anthemideae): New data on systematics and phylogeography. Willdenowia 36: 69-87.

El-Shazly, A. M., S. S. Hafez and M. Wink. 2004. Comparative study of the essential oils and extracts of Achillea fragrantissima (Forssk.) Sch Bip. and Achillea santolina L. (Asteraceae) from Egypt. Pharmazie 59: 226-230.

Guo, Y.-P., J. Saukel, R. Mittermayr and F. Ehrendorfer. 2005. AFLP analyses demonstrate genetic divergence, hybridization, and multiple polyploidization in the evolution of Achillea (Asteraceae-Anthemideae). New Phytologist 166: 273290.

Hara, H. 1938. Preliminary report on the Flora of southern Hidaka, Hokkaido (Yezo) XXIV. Botanical Magazine Tokyo 52: 1-8.

Im, R. J. 1999. Flora Coreana Vol. 7 (Asteraceae). The Science and Technology Publishing House, Pyongyang. Pp. 136-145.

Koyama, H. 1995. Asteraceae (Compositae). In Flora of Japan, IIIb, Angiospermae, Dicotyledoneae, Sympetalae (b). Iwatsuki, K., T. Yamazaki, D. E. Boufford and H. Ohba (eds.), Kodansha, Tokyo. Pp. 77-78.

Kitamura, S. 1968. Compositae of Southeast Asia and Himalayas I (in Japan). Acta Phytotaxonomica et Geobotanica 22: 1-19.

Koidzumi, G. 1918. Contributiones ad florum Asiae orientalis (Continued from Vol. XXXI). Botanical Magazine Tokyo 32: 53-63.

Saukel, J., M. Ancev, Y. P. Guo, A. Vitkova, A. Nedelcheva, V. Goranavo, A. Konakchiev, M. Lambrou, S. Nejati, F. Rauchensteiner and F. Ehrendorfer. 2003. Comments on biosystematics of Achillea (Asteraceae-Anthemideae) in Bulgaria. Phytologia Balcanica 9: 361-400. 